\title{
Una torre en la muralla de Biar. Consolidación y recuperación de una imagen urbana
}

A tower in the wall of Biar. Consolidation and recovery of an urban image

\author{
Miguel del Rey ${ }^{a}$, Antonio Gallud $^{\mathrm{b}}$, Silvia Bronchales ${ }^{\mathrm{c}}$ \\ Instituto Universitario de Restauración del Patrimonio - Universitat Politècnica de València, Valencia, Spain \\ a juanmigueldelrey@gmail.com; ${ }^{\mathrm{b}}$ agallud@upv.es; ' ${ }^{\mathrm{s}}$ silbroal@pra.upv.es
}

\begin{abstract}
The wall of Biar goes around the historical part of the city and connects it with the castle at the top of the hill. This urban wall was comprised by the city wall and a walk wall, which were protected by a battlement and a series of towers. Currently, the urban wall has been swallowed by changes in the area. Internal edifications to the city wall have progressively taken over the wall and, in its outside part, an area as wide as the towers has been occupied, which has eventually set up the front part of Torreta street. The Tower of Jesus is part of this defensive set that nowadays is almost invisible.

Before its restoration, the tower was in an unfortunate state of abandonment and deterioration. Large cracks in its masonry warned of its immediate collapse. After its defensive use, it was transformed and joined to more modern neighboring buildings. Removed walls, deformed gaps and variations in the roof concealed its past as it went unnoticed and passed as another house on the street. Only the traces in its walls exposed its history.

The intervention process for its recovery began with a thorough, formal, dimensional and technical study, to subsequently propose its restoration and the choice of contiguous elements that had to be eliminated to show a recognizable set. Also, a new way of walking and using it was put forward. During the intervention, several objectives were considered. In addition to the most obvious ones, such as the structural consolidation that would prevent its eventual collapse, recovering its historical image and showing the key facts that would lead to interpreting its past and discovering its secrets. Besides describing in detail the restoration process in its entirety, this text aims to present the issues that were raised during the intervention and to consider those reasons behind all the decisions that were made.
\end{abstract}

Keywords: Tower of Jesus, Biar, urban wall, restoration.

\section{Introducción}

El castillo de Biar en la provincia de Alicante se sitúa en lo alto del Cerro en posición dominante en el territorio. El núcleo urbano de Biar se asienta en su cara sur la más amable por orientación y pendiente, al amparo del Castillo. El sistema del que forma parte el Castillo esta configurado por la torre celóquia y una doble muralla que da forma al patio de armas más una tercera muralla que, bajando por la ladera, envuelve la ciudad histórica atándola a la fortaleza defensiva. Tanto en el cerro como en la propia ciudad histórica se pueden encontrar y seguir varios restos que indican el antiguo trazado de la muralla que rodea la ciudad, actualmente estos restos precisan de un estudio en profundidad y un trabajo de recuperación que los ponga en valor y de 
esta forma ofrezca una lectura más clara de la historia de Biar, además de evitar que acaben desapareciendo.

El centro histórico mantiene, en buena parte, el parcelario y trazado medieval, se organiza a base de calles longitudinales ligeramente que mantienen la cota del terreno en su recorrido y las calles transversales con gran pendiente y con el castillo como fondo visual.

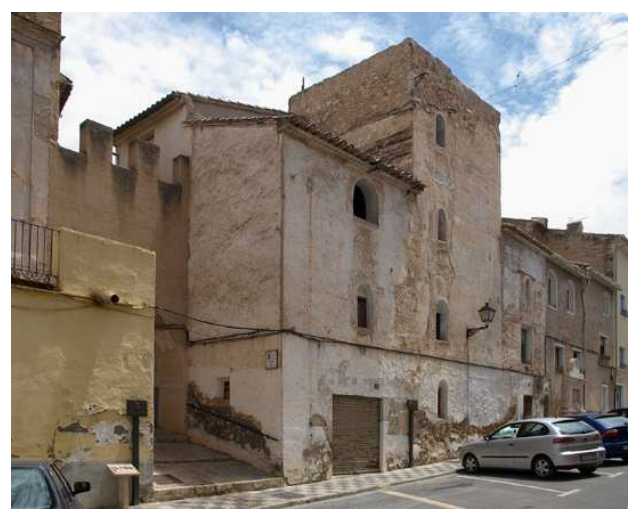

Fig.1. Imagen de la Torre de Jesús previa a la intervención (Miguel del Rey, 2009).

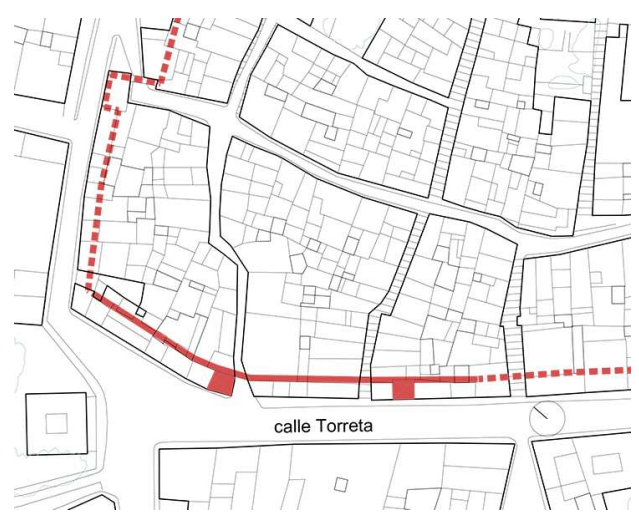

Fig.2. Hipótesis del trazado de la muralla urbana de Biar (elaboración propia, 2009).

El trazado de la muralla que encerraba el centro histórico estaba constituida por el propio muro de muralla con adarve protegido por almenas y una serie de torres que pautaban la misma.

Esta muralla urbana, con el tiempo, ha sido engullida y en la actualidad forma parte del parcelario. En la cara interior de la muralla las edifi- caciones adosadas a ella han ido ampliando su espacio a través de un proceso de apropiación, excavación y ocupación en forma de alacenas, armariadas y oquedades en el espesor del muro, mientras que al exterior los espacios que existían entre las distintas torres han sido edificados tanto apoyándose en el lienzo de muralla como en el muro posterior de apoyo, y manteniendo como frente de fachada la cara principal de las torres. Un claro ejemplo es la calle Torreta, donde su frente norte el resultado de esa transformación, mientras su frente sur es fruto de posteriores ensanches de la ciudad.

\section{La Torre de Jesús}

\subsection{Descripción de la Torreta}

En la calle Torreta y junto a la puerta de Jesús se encuentra uno de los pocos restos que se mantienen del sistema defensivo urbano de Biar, es la Torre de Jesús, la denominada "Torreta", que da nombre a la calle. La torre defensiva se encuentra oculta en la actualidad a una rápida visión del conjunto ya que a ambos lados de la misma se levantan dos edificios que ocultan las caras laterales de la torre y la muralla, aunque con transformaciones, añadidos y mutilaciones que distorsionan su imagen y aminoran el valor de la torre como elemento emergente y vinculado a la muralla de la que forma parte.

La torre tiene planta casi cuadrada de 5,30 x $5 \mathrm{~m}$ y una altura de $12 \mathrm{~m}$, ha estado abandonada en los últimos años, acelerando su proceso de deterioro. Hasta mediados del siglo XX fue usada como vivienda y taller unida a los edificios anexos, formando una unidad, de modo que los tres edificios se enlazaban internamente horizontal y verticalmente en un intrincado laberinto de pasos, escaleras y particiones que han ido variando en el tiempo.

La Torre es un edificio prismático vertical de fábricas portantes perimetrales construidas de muro tapial, apoyado directamente sobre un pequeño basamento de mampostería que apoya sobre la roca. El nivel de asiento ha sido excavado y vaciado formando un muro de roca emergente desde el nivel actual de la calle exterior, quedando al aire la unión de roca y nacimiento del muro, lo cual queda en algunos casos a más de 
$125 \mathrm{~cm}$ del plano actual de la calle. Las excavaciones se han sistematizado en el interior de la torre, vaciando el nivel de cimentación original.

\subsection{Estructura de la Torreta}

La torre tiene una estructura portante particular, ya que en algunos niveles no desaparece el muro interno, utilizando como portantes los tres lados externos a límite de la muralla. Dando la impresión que fuera la propia muralla la que se plegara definiendo así la torre, a la manera de cómo están resueltas las torres en la zona islámica del propio Castillo de Biar, y como era práctica corriente en la época.
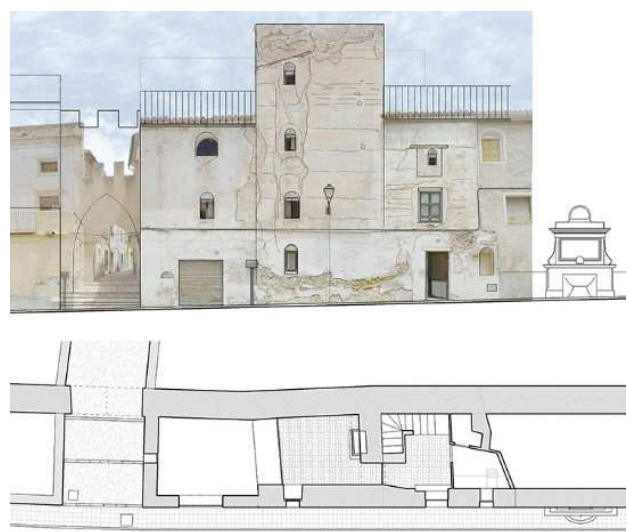

Fig. 3. La Torreta previa a la intervención (2009).

En la actualidad existen cuatro niveles interiores en la torre, resueltos con forjados muy diversos apoyados de manera distinta en cada planta. Los forjados originales se sitúan en los niveles superiores, mientras que el forjado inferior es de factura moderna, e incluso relativamente reciente. Los forjados superiores se disponen alternativamente en paralelo y perpendicular a la línea de muralla y se construyen con vigas de gran escuadría y revoltones cortos. El forjado inferior es de escuadría muy débil, su dirección es perpendicular a la calle y está apoyado a escasos $80 \mathrm{~cm}$ del inicio del muro de tapial en el muro de fachada y sobre la línea de carga de la muralla en la parte contraria.

La particular arquitectura consta de un sistema de muros de gravedad de los cuales los portantes son los que se definen como un pliegue perime- tral externo de la muralla la cual solo traba en la cimentación y en el nivel tres actual de la torre.

\subsection{Afecciones Estructurales}
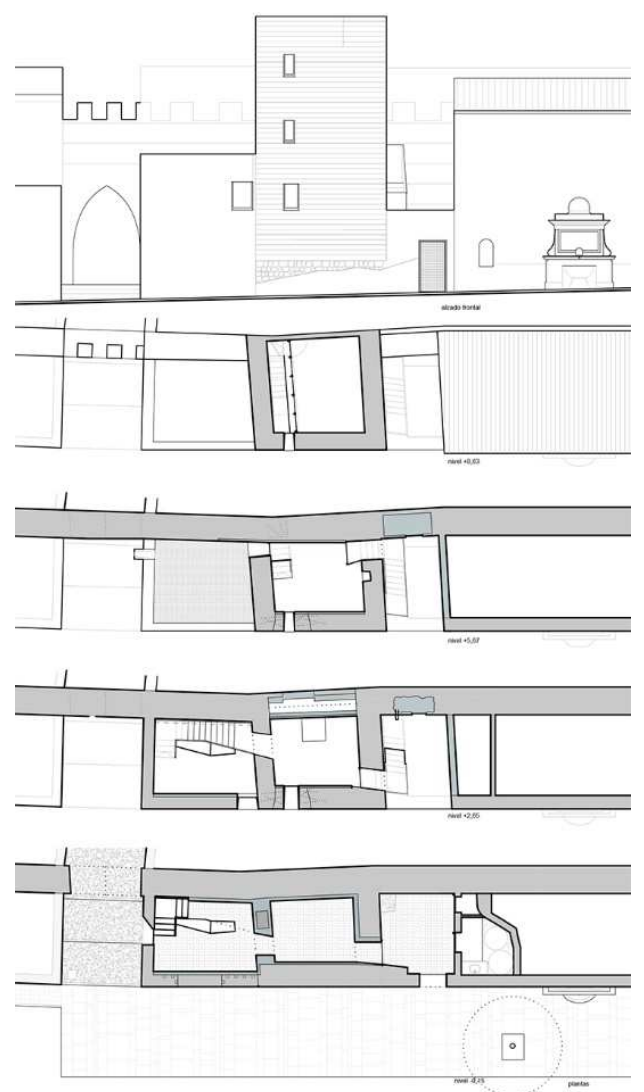

Fig. 4. Plantas y alzado de la intervención (2018).

En la historia reciente el muro inferior de apoyo de la torre en el lado perpendicular izquierdo, mirando desde la calle, ha sido demolido, para de esta forma unir la planta baja del cuerpo anexo y la planta inferior de la torre. Esta planta baja de la torre a su vez ha sido excavada, para ganar espacio en el interior, bajando el nivel original de la torre y dejando al aire la unión del muro con la roca virgen, al recortar por ambos lados: por el exterior, para nivelar la calle, y por el interior para sacar altura suficiente para usar como planta un nivel que no existió en origen.

La demolición del muro transversal ha supuesto una gran reestructuración de los esfuerzos en la 
torre, de forma que se generaron deformaciones en forma de grandes flechas en el vano de paso entre ambos elementos así como en el propio forjado, pero sobre todo ha generado un reequilibrado de la estructura muraría reflejada en unas grandes grietas en la parte más débil del muro de la torre, donde se sitúan verticalmente las ventanas del muro de fachada, colindante con el muro eliminado, generando la rotura de la traba en la esquina del tapial, separándose ambos muros y apareciendo en el propio ángulo una gran grieta vertical visible desde la fachada que corre desde arriba a abajo entre las ventanas. Grieta que se amplia y zigzaguea englobando ambos lados de los huecos de las ventanas.

Como resultado de todo ello, la estructura portante quedó muy dañada y aparecieron una serie de grietas preocupantes que muestran la dureza del reequilibrio estático; reequilibrio que agrietó fuertemente el muro de fachada e hizo improbable la estabilidad por sí sola de la torre.

\section{La Propuesta de Intervención}

\subsection{Propuesta arquitectónica y urbana}

La propuesta arquitectónica y urbana están ligadas, ambas pretenden poner en valor al conjunto de la Torre y la Muralla como elemento significativo dentro del patrimonio arquitectónico constructivo y paisajístico de Biar en un contexto absolutamente diferente del que dio origen a su construcción. La absorción e integración dentro de la trama urbana consolidada es un hecho irreversible. La propuesta trata de recuperar y evidenciar sus valores arquitectónicos dentro de su propio contexto urbano actual, se pretende recuperar la silueta de la torre y resaltarla por su volumetría como elemento emergente a la muralla y por el carácter y textura de sus fábricas frente a los edificios colindantes.

\subsection{Estudios previos}

Previo a la propuesta arquitectónica de la Torreta se procedió a un estudio exhaustivo de su morfología, dimensiones, evolución historia, proceso urbano, materialidad, técnicas constructivas, etc. con el objetivo de tener conocimiento del porqué de su estado actual y cual sería la intervención más adecuada y acorde al valor del elemento patrimonial y su contexto. Así mismo, previo a la intervención, se realizó un estudio arqueológico estratigráfico que ayudó a verificar los datos de los estudios y observaciones y corroboró las decisiones de intervención previstas.

\subsection{Propuestas de eliminación de elementos}

Con toda la información recopilada el paso siguiente, ya como parte de la intervención, fue eliminar los elementos añadidos con el paso del tiempo que alteraban la torre.

Los actuales edificios colindantes a la torre son de distinta naturaleza y afectan de distinta manera a la torre, el objetivo de la intervención urbana que se persigue es recuperar la imagen de la torre en continuidad con la muralla oculta tras las edificaciones contiguas. Si bien la decisión más obvia sería su eliminación, se optó por una actitud más reflexiva que ponía en valor no solo el momento primigenio sino pasajes posteriores compatibles con el objetivo marcado en la intervención, de este modo se decidió rebajar una planta más la cubierta el edificio colindante $n^{\circ}$ 20 de la calle Torreta y eliminar en su totalidad el del $n^{\circ} 18$ dejando el muro de fachada en planta baja a modo de tapia tras la que se genera un patio de acceso desde el que se puede observar el lateral de la torre en su totalidad y del lienzo de muralla, recuperando la imagen completa desde cimentación hasta remate de cubierta.

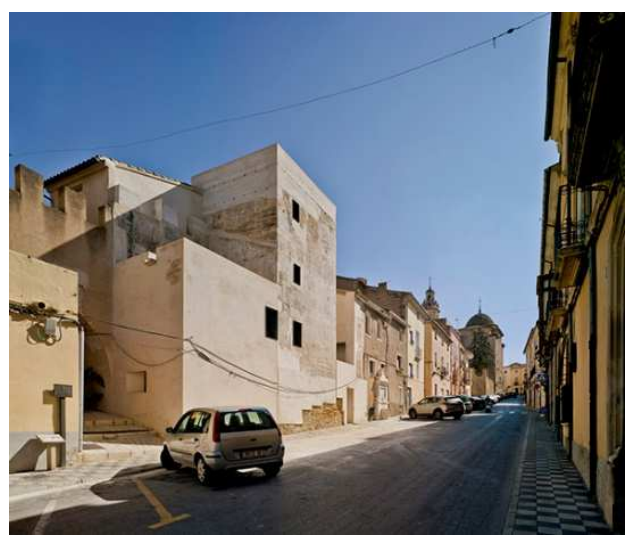

Fig. 5. Torreta, estado tras la intervención (David Frutos, 2018). 
El nivel eliminado del $n^{\circ} 20$, fue le más significativo ya que ocultaba el lienzo de la muralla con una serie de almenas en continuidad con las de la puerta de Jesús, hoy ocultas. Respecto a las plantas inferiores era aconsejable mantenerlas desde el punto de vista estructural ya que servían de apoyo lateral a la torre. El hecho de rebajar la edificación anexa en este lateral conseguía un efecto potenciador en la altura de la torre y permitía la lectura buscada de la continuidad entre la torre y la muralla.

\subsection{Organización interior previa}

La planta inferior de la torre presenta una naturaleza aparentemente confusa, parte de ella está formada por roca natural tallada a modo de muro, mientras que a media altura se inicia el muro de tapial, indicio de que la planta baja es fruto de una excavación posterior al uso defensivo de la torre.

Respecto a la propia torre, el edificio conserva su característica histórica y mantiene la continuidad de uso que ha tenido en los últimos tres siglos, junto a los edificios colindantes; en este momento manteniendo la autonomía de sus diversas arquitecturas. Se conserva el acceso a través del anexo derecho de la torre, por medio de una cancela que permite la vista del patio interno, desde el cual se pasa al nivel inferior de la torre y por allí al resto. Patio que incluye los servicios higiénicos y un pequeño almacén.

\subsection{Intervención estructural}

Estructuralmente, la intervención pretende estabilizar la estructura muraria que funciona por gravedad, rellenar y sellar las grietas y reequilibrar muros y la propia geometría portante. La intervención estructural consistió en reforzar la parte de la base de la Torre que se encontraba en muy precaria situación, recuperar lienzos de muro inexistentes eliminados en su totalidad y recrecer los debilitados hasta generar una geometría estable y lógica.

Las caras interiores de los muros de la torre estaban muy deteriorados, los tapiales tenían grandes faltas y rebajes generalizados. En la intervención las estructuras verticales se consolidaron trasdosando en una de las caras láminas de hormigón de cal con arena de la zona de distintos espesores 16 o $10 \mathrm{~cm}$, ancladas con varillas de acero a la base existente. Muros encofrados para ser vistos con listones de $22 \mathrm{~cm}$ en algunas de las caras, siguiendo la métrica de los muros de tapial originales.

De entre los muros eliminados históricamente, el más preocupante era el de medianería con el edificio $\mathrm{n}^{\circ} 20$, eliminado en planta baja de la torre. Su reposición se hizo en dos fases. En la primera, provisional dada la precariedad de la situación, se construyeron dos machones de ladrillo panal, acabando los machones en su cabeza con hormigón. Estos machones se dispusieron de manera que uno de ellos quedará incluido en el propio muro de hormigón que más tarde se construyó, mientras que el otro se dispone de manera que permita el paso por medio de ambos. Una vez hormigonadas las láminas trasdosadas de los muros que sustituyen al muro desaparecido, se eliminó el último de los machones de ladrillo, mientras que el primero queda embebido dentro de su masa.

Las láminas de hormigón encofradas con listonado de $22 \mathrm{~cm}$ se levantaron hasta la planta primera de la torre en alguna de sus caras, trasdosándose a los tapiales unidos por espadas que penetran en ellos, perforadas por taladros en los que se incluye una barra de acero y se inyecta mortero de cal de alta adherencia. La lámina interna de la fachada, de menor espesor, se prolongó en las plantas superiores y se utilizó para estabilizar y atar la propia fábrica y las numerosas y preocupantes grietas verticales existentes.

Las grietas en los muros de la fachada se estabilizaron con un cosido desde la lámina trasdosada de hormigón con cal encofrada con listones de $22 \mathrm{~cm}$ de ancho y $10 \mathrm{~cm}$ de espesor, levantada por tongadas de un tercio de altura de entre planta; muro unido por medio de conectores de acero de $6 \mathrm{~mm}$ y profundidad variable, mínimo $30 \mathrm{~cm}$ cada $50 \mathrm{~cm}$ en horizontal y vertical, y grapas de unión y atado de grietas selladas con un mortero de cal de alta adherencia. En el caso de las grietas inmediatas a las ventanas, se cosieron desde las jambas de los huecos. Una vez finalizadas estas operaciones de estabilización estructural se sellaron las grietas con un mortero de cal. 
La cubierta de teja sobre forjado de rollizos de madera y el muro de mampostería existente en la Torre se eliminó y sustituyó por una solución más propia de este tipo de torres, una cubierta plana en el último nivel, enrasada al nivel coincidente con el adarve de la muralla encontrado durante la obra.

Los huecos existentes tenían un carácter más domestico que defensivo, eran el resultado de una transformación reciente de la fachada. No se encontraron indicios de los huecos originales, por lo que se optó por cerrar los huecos que se podía apreciar que se habían generado de forma reciente y se ordenó los que se mantienen en forma y tamaño aplicando las normas lógicas de la construcción del tapial.

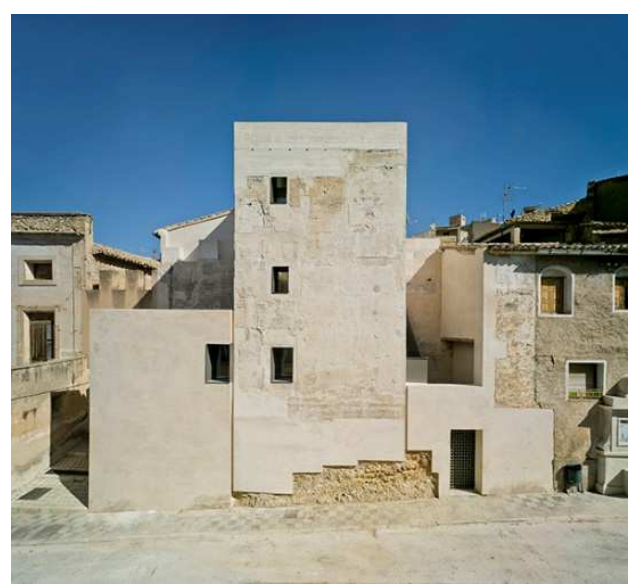

Fig. 6. Torreta, estado tras la intervención (David Frutos, 2018).

\subsection{Intervención arquitectónica}

Desde el punto de vista de la propuesta arquitectónica, parece conveniente destacar el criterio metodológico aplicado y consistente en restaurar todo aquello que nos ha legado la historia y se dispone de información suficiente para ello, evitando el recurso de los falsos historicismos, y utilizar en las intervenciones de nueva planta materiales y lenguajes actuales, que armonicen con el contexto en el que se integran y potencien, a su vez, todo aquello que arquitectónica e históricamente resulte de interés.

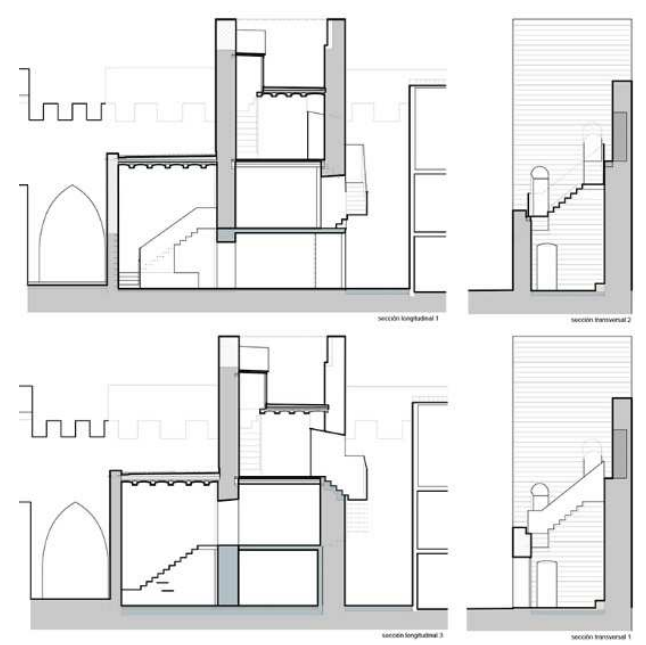

Fig. 7. Secciones de la intervención (2013).

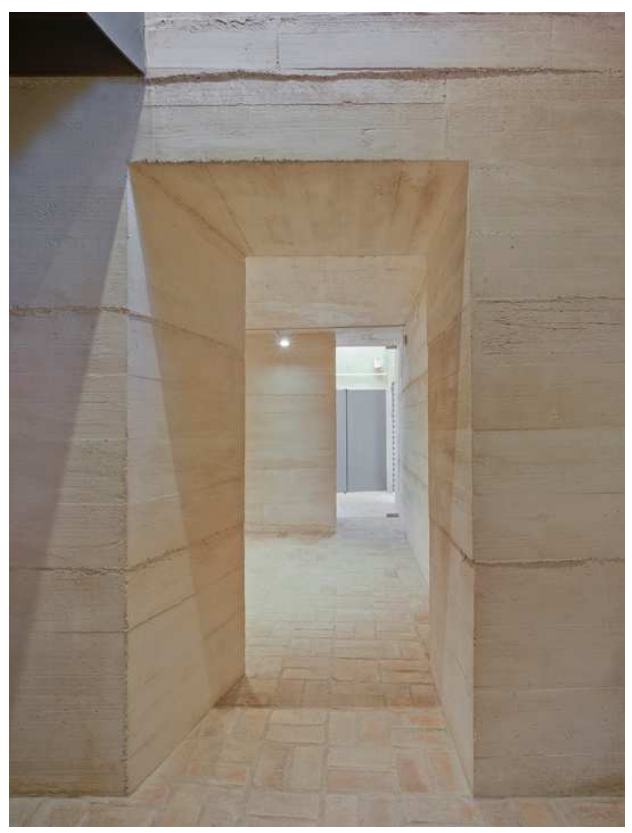

Fig. 9. Interior de la Torre (David Frutos, 2018).

Desde el inicio de la intervención del proceso de intervención se planteó la necesidad de dar utilidad al edificio, para ello era necesario dotarlo de unas condiciones mínimas de habitabilidad que, evidentemente, no perjudicarán la lectura del bien a restaurar. La estrategia utilizada fue construir una escalera que al subir por ella produjera un desplazamiento que obligara a recorrer el edi- 
ficio por múltiples dimensiones y, a modo de observatorio, se desplegara por el proyecto, entrando y saliendo de los espacios y permitiendo, por ejemplo, recorrer la torre por el exterior y tener contacto con las caras exteriores en niveles elevados y, hasta ahora, inaccesibles. La escalera se construyó como un elemento autónomo estructural y materialmente. Es una estructura rígida de plancha de acero que parece no tocar la estructura de la torre. El proyecto arquitectónico se gesta como un recorrido, como un nuevo modo de habitar la Torreta.

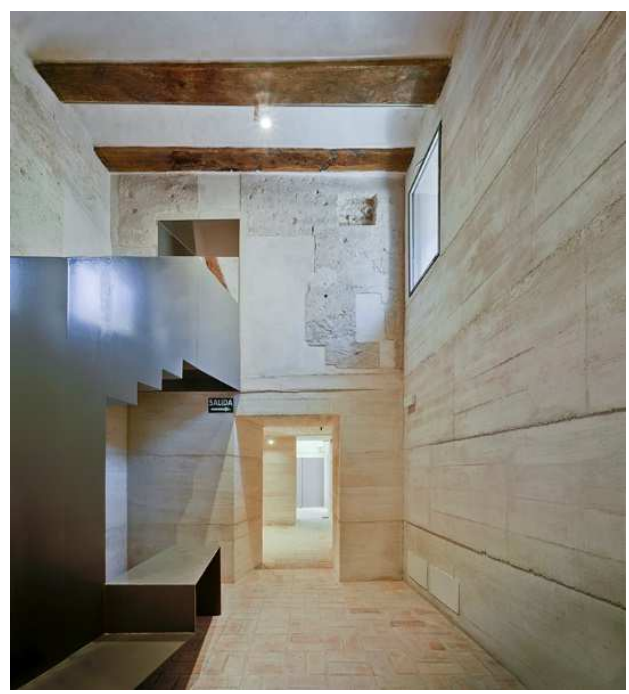

Fig. 10. Torreta, estado tras la intervención (David Frutos, 2018).

Desde el patio se puede divisar la totalidad de la altura de la Torre y su textura. Una puerta de una hoja de vidrio sin marco superpuesta al muro permite la entrada al nivel inferior de la torre y atravesando esta estancia, de uso expositivo se pasa hasta la planta a nivel del suelo del edificio $\mathrm{n}^{\circ} 20$, donde se dispone la atención al público y una escalera paralela a la muralla conduce al nivel superior de la torre recorriendo un espacio a doble altura con una luz termal. A través de una puerta abocinada se accede a la torre en su nivel principal y donde por un hueco se sale al patio donde podemos asomarnos y ascender por una escalera exterior separada del muro desde la que se puede apreciar la textura de las fábricas originales y llegar hasta el nivel superior. En esta úl- tima sala se encuentra una pequeña hornacina descubierta durante el proceso de intervención, un cierre ocultaba un tesoro olvidado al que las leyendas locales hacían mención, aunque en vez de joyas el tesoro estaba compuesto por objetos cotidianos, pinturas religiosas y mapas de gran valor histórico y documental que hablaban de conexiones pasadas con lugares lejanos. Será necesario un estudio minucioso que relate las vidas de los que ocultaron esos objetos. Desde esta planta una escalera de yeso preexistente permite acceder a cubierta donde se puede observar el paso de guardia de la muralla y el volumen recuperado de la torre.

\section{Conclusiones}

A través de un proceso de estabilización estructural muy complejo debido al estado tan delicado en que se encontraban las fabricas antes de la actuación se ha ido recuperando la memoria olvidada y casi perdida de la Torreta. Se ha conseguido estabilizar la estructura de la torre, se ha recuperado para uso público el disfrute de este bien público y se ha recuperado la memoria de elemento de arquitectura defensiva sobre el lienzo de la muralla como parte de un todo mucho más amplio por descubrir.

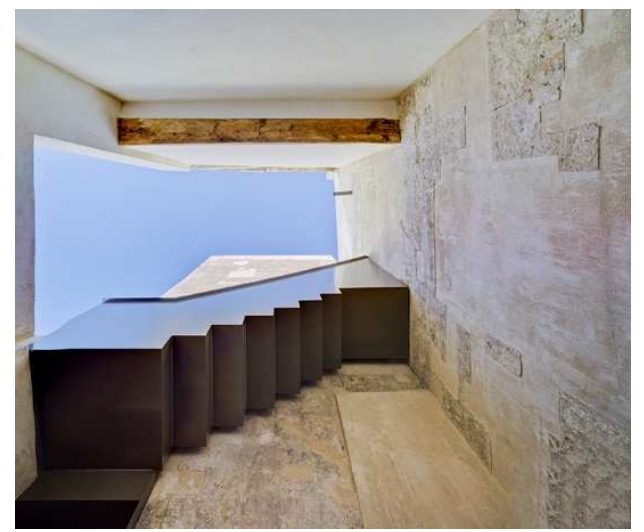

Fig. 8. Patio de Acceso a la Torreta (David Frutos, 2018).

Se ha recuperado su presencia en el paisaje urbano de la localidad como torre y parte de un sistema defensivo de la ciudad, devolviendo la silueta rotunda y másica que la torre generaba en 
el lienzo de la línea de muralla, haciéndola reconocible.

Se ha recuperado su volumen original, la conexión con el paño de muralla y con el adarve de la misma y las almenas de la parte izquierda que en un futuro esperanzador continuaran con los lienzos del arco de Jesús y del edificio colindante hoy ocultas tras revocos y otros muros.

Se ha recuperado la torre elemento protector antaño, como espacio cultural que ayude a mantener el origen del asentamiento y su evolución así como los distintos abatares hasta los momentos actuales.

Se han recuperado las texturas, las trazas, las huellas y cicatrices de las fábricas de tapia, dejando que ellas mismas relaten su historia vivida.

La mirada cercana que se genera sobre el volumen de la torre recuperada y sobre las texturas distintas de la tapia que ahora aparecen y se descubren antes ocultas por revocos, grietas y suciedad, nos devuelven igual que un libro abierto páginas de historias escritas.

\section{Bibliography}

Azuar, R. (1983). "Panorama de la arqueología medieval de los valles alto y medio del Vinalopó (Alicante)", Lucentum, 2, pp. 349-383.

Bérchez, J.; et al. (1983). Catálogo de monumentos y conjuntos de la Comunidad Valenciana, Consellería de Cultura, Educación y Ciencia, Generalitat Valenciana Ed., Valencia.

Brandi, C. (1999). Teoría de la restauración, Alianza Ed., Madrid.

Capitel, A. (1988). Metamorfosis de monumentos y teorías de la restauración, Alianza Ed., Madrid.

Font, F.; Hidalgo, P. (1990). El tapial: una técnica constructiva milenaria, Colegio Oficial de Aparejadores y Arquitectos Técnicos de Castellón Ed., Castellón.

Guinot, E. (1995). Els límits del Regne: el procés de formació territorial del País Valencià medieval (1238-1500), Alfons el Magnànim Ed., València.

Rey, M. Del.; Gallud, A. (2011). Intervención y consolidación del segundo recinto amurallado del Castillo de Biar, UPV Ed., Valencia.

Segura, G.; Simón, J.L. (2001). "Las murallas medievales de Villena”, in Castillos y torres en el Vinalopó. Col-leecció l'Algoleja, 4, Centre d'Estudis Locals del Vinalopó Ed., Petrer, pp. 83-88.

Torró, J. (2012). "La conquista del reino de Valencia. Un proceso de colonización medieval desde la arqueología del territorio", in La conquista de Al-andalus en el siglo XIII, Centro de Estudios Medievales Ed., Murcia. 